\title{
Experimental study on performance of high purity nitrogen supply system in liquefaction unit of low concentration coalbed methane
}

\author{
JIA Xiao-liang ${ }^{1, \mathrm{a}}$ \\ ${ }^{1}$ Chongqing Research Institute Co, Ltd. China
}

\begin{abstract}
Aiming at the problems such as low CBM utilization rate caused by low concentration CBM with methane content less than $8 \%$ extracted from a small coal mine in Hancheng mining area, Shaanxi Province, and serious environmental pollution caused by coal-fired hot blast stove for heating, the heat storage and oxidation heating technology of ultra-low concentration gas in small coal mine was studied by combining field investigation and heat balance calculation. This technology replaces the coal-fired hot blast stove, realizes "coal and CBM mining and sharing" in the coal mine, and constructs a virtuous cycle development of "promoting drainage by using and promoting safety by drainage". The research shows that: the scheme improves the utilization rate of CBM in the mining area, cancels the consumption of coal combustion, and can use 2.59 million Nm3 of pure CBM every year, generating 1.95 million yuan of economic benefits. And the emissions reduction of $\mathrm{CO} 2$ equivalent is 36000 tons. The benefits of safety, economy and environmental protection are remarkable. This technology is especially suitable for the utilization of ultra-low concentration $\mathrm{CBM}$ whose concentration is less than $8 \%$, which has practical significance to improve the CBM utilization rate and promote the realization of the goal of "zero emission".
\end{abstract}

\section{Introduction}

Low concentration of coal seam gas deep cold liquefatis is a new technology in the development and utilization of coal seam gas in coal mining areas, in which mixed-quality refrigeration is an energy-efficient refrigeration process ${ }^{[1-4]}$. Mixed refrigerant refrigeration uses 3 temperature zones, of which the cold in the low temperature zone is mainly derived from the thwart of nitrogen ${ }^{[5]}$. In the coal seam gas deep-cooled liquefaction device, nitrogen is mainly separated from the air by variable pressure adsorption method, and the variable pressure adsorption nitrogen device is very important for the control of the purity of nitrogen products ${ }^{[6]}$. If the nitrogen purity is not up to standard, the mixing refrigerant channel in the cold box creates an "ice block" that increases power consumption and stops the unit for long-term operation ${ }^{[7-8]}$.

\section{2 experiment procedure}

Figure 1 provides the laboratory with a dual adsorption column variable pressure adsorption nitrogen production device with a purity of more than $99 \%$. Ambient air first removes solid impurities through filter 1 , then is sucked into screw compressor 2, compresses the gas to adsorption pressure, then cools the compressed air to room temperature by cold dryer 3, and removes condensed moisture, due to molecular sieve to water Strong adsorption, so can not let water into the main reaction adsorption column, reduce the adsorption performance of the molecular sieve, while the carbon molecular sieve is a polar compound, the air carbon dioxide, carbon monoxide, acid and ozone and other polar molecules adsorption capacity is very strong, These substances should be removed before entering the adsorption column, the experimental device through filter 4 to remove the gas saturated water, oil, carbon dioxide and other impurities, at this time the gas flowing out can be regarded as oxygen, nitrogen mixture of two parts, the mixture through the flow meter to measure the air flow into the main reaction adsorption column 9, PLC control system issued instructions, so that the solenoid valve switch, 2 adsorption column cycle for adsorption and regeneration, to achieve the purpose of continuous product. Product nitrogen through the nitrogen meter 8, flow meter 7 into the nitrogen tank 10 .

The air intake and venting of adsorption column and the whole process of adsorption system are controlled by solenoid valve group and time control system. The solenoid valve can be divided into two parts: the electromagnetic control part and the pneumatic valve. The solenoid valve controls the pull rod through the action of the electromagnetic force, and drives the pull rod action through the action of the air pressure, so as to achieve the purpose of closing or opening the valve, and

ae-mail:2010041@cqccteg.com 
control each step of the process flow of the system. The time control system of the device is controlled by PLC.

It can be seen from Figure 2 that the working steps of the adsorption column of this experimental device include 8 , and the time of each step is represented by T1 T8 respectively. There are 7 valves controlling each step, which are $\mathrm{V} 1 \sim \mathrm{V} 7$. In the process of debugging, you only need to modify the time of the corresponding steps in the program, which is very convenient. Combined with figure 1, the working process of PLC is briefly described. As shown in the figure, M0 starts, V1, V4 and V5 lines are connected, and the valve is opened. At the same time, T1 starts timing. After T1 timing, T1 acts, then V7 valve line is connected, V7 valve is opened. When T2 timing is finished, T2 acts, then V4 valve line is disconnected, and V4 valve is closed. And so on, each step in turn.

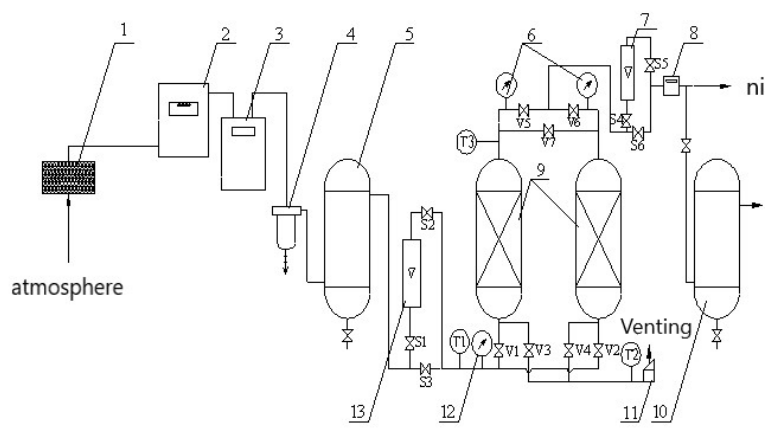

Figure1 A general diagram of the flow of the experimental device

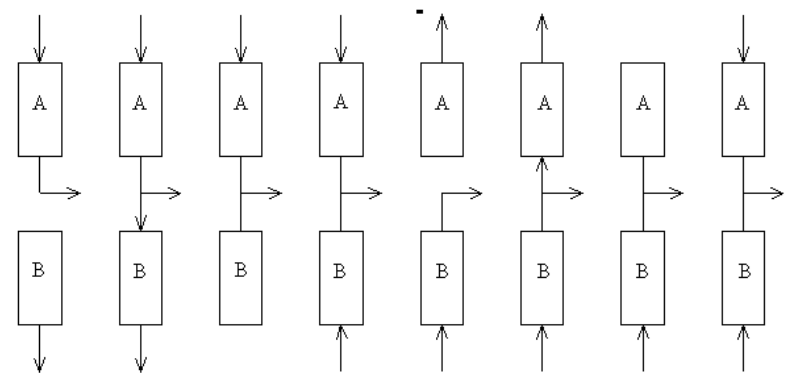

Figure 2 Schematic diagram of PSA cycle process

\section{Analysis and research of experimental results}

Figure 2 shows the adsorption pressure, blow-back time, the mean pressure time is fixed, different raw material air inflection conditions, adsorption time on nitrogen production performance, the horizontal coordinates in the figure are relative adsorption time. As you can see from the figure:

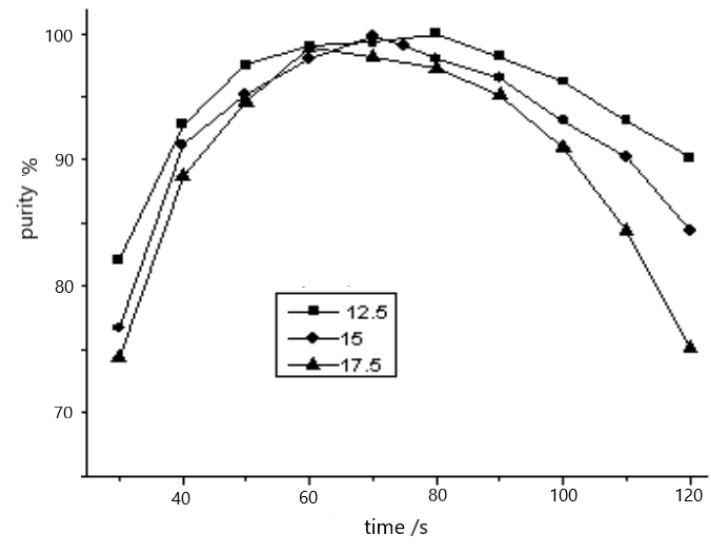

Figure 3 The effect of adsorption time on product purity at different air inflection flows

(1) Under the same air flow conditions, the adsorption time increases and the purity of the product increases rapidly. When purity increases to peak, it begins to decrease with the increase in adsorption time, and there is an optimal adsorption time. When the adsorption time increases, the adsorption pressure of the molecular sieve in the adsorption column increases, so that the adsorption of the molecular sieve on the oxygen part increases. On the other hand, the adsorption time increases and the adsorption column regeneration in the regeneration stage will be more thorough. Therefore, at the beginning of adsorption, the purity and yield of the product increase significantly with the increase of adsorption time. As the adsorption process continues, the raw material gas flow exceeds the saturation capacity of the carbon molecular sieve in the adsorption column. After adsorption saturation, oxygen as a carbon molecular sieve strong adsorption part, penetrate the adsorption bed layer (i.e. oxygen leakage), product purity decreased. Therefore, for a particular condition, there is an optimal adsorption time.

(2) The greater the intake, the shorter the adsorption time for the best purity. The adsorption of oxygen and nitrogen on the carbon molecular sieve is balanced adsorption, and the axial air speed of the adsorbent bed is very small relative to the mass transfer rate. However, the speed of movement of the frontier of the mass transmission region is related to the amount of air intake, the greater the amount of intake, the faster the movement speed of the fluid phase in the adsorption column, thus speeding up the movement speed of the frontier of the mass transmission zone. That is, under certain adsorption bed height conditions, the higher the air flow, the shorter the optimum adsorption time. However, it can be seen from the figure that increasing the intake volume will affect the purity of the product.

\subsection{Effect of operating pressure on adsorption performance}

Operating pressure is an important factor affecting the separation performance of variable pressure adsorption. When the carbon molecule sieve filling amount (i.e. adsorption bed size) is determined, the operating pressure 
can be increased, which can increase the adsorption capacity of the adsorption phase (oxygen) on the carbon molecule sieve, which is conducive to the separation of nitrogen and oxygen parts. In addition, different adsorbents on the operating pressure requirements are different, the actual operation pressure and carbon molecular sieve the best operating pressure is closer, the better the effect of gas separation. Secondly, the comprehensive energy consumption of the variable pressure adsorption device is related to the operating pressure, which increases the operating pressure and increases the comprehensive energy consumption. For variable pressure adsorption nitrogen system, the higher the operating pressure, the smaller the compressor displacement and the greater the noise. Higher operating pressures also increase the temperature of the compressor outlet gas, burdening the cooling system. Therefore, in the case of ensuring adsorption effect, the operating pressure should be reduced as much as possible. It is of great significance to study the operation pressure and related process parameters of variable pressure adsorption nitrogen device to further improve the separation effect of the equipment. Figure 3 shows the effect of operating pressure on the purity of nitrogen products and the effect of blow-back time on operating pressure. The conclusions are as follows:

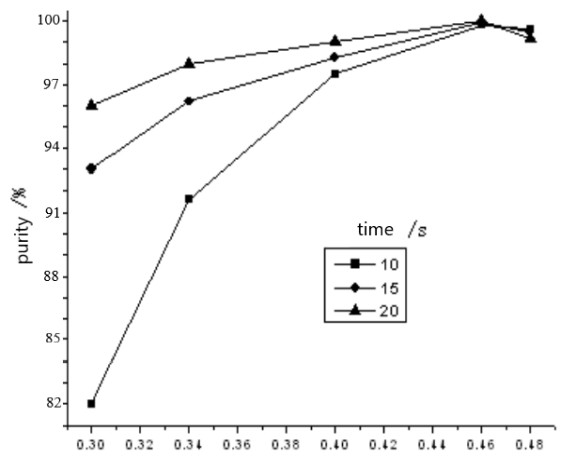

Figure 4 Effect of operating pressure on product purity at different blow-back times.

\subsection{Effect of average pressure time on adsorption performance}

As can be known from the mass transmission zone model of variable pressure adsorption, the adsorption bed layer is divided into adsorption saturation zone, mass transmission area and unsorted zone from the air infrasortation end to the air exhaust end, wherein the gas between the molecular sieve voids in the adsorption saturation zone (note that it is not pores) is high pressure. The unsorted clean raw gas, the gas in the mass transmission area is a mixture of nitrogen-rich gas and air, the nitrogen purity of the gas is between the raw material air and product purity, the gas between the molecular sieve gaps in the unsaturated zone is a product gas with high nitrogen purity, in practice, The adsorption process should be moved to the front of the mass transmission area before the suction bed exit, and a certain amount of operating balance should be left to prevent oxygen penetration, resulting in product contamination. If the open empty valve is opened at the end of the adsorption for reverse emptying, the adsorption saturation zone and the adsorption bed dead space (cannot fill the space of the molecular sieve, such as the space around the air flow distribution and the space of the pipeline) of the pressurized air and unsorted area of the nitrogen will be white discharged into the atmosphere, resulting in waste. Therefore, the variable pressure adsorption process usually introduces the average pressure step.

Figure 4 shows the effect of the average pressure time on the purity of the product, and it can be found from the figure that with the increase of the average pressure time, the purity of the product increases to the peak and then decreases gradually, and the optimal average pressure time exists. In a particular device, after the average pressure, the gas in the edgy adsorption column is replenished, so that the operating pressure is raised rapidly to ensure the purity of the outlet nitrogen. However, when the average pressure time reaches a certain value, the oxygen de-absorbed by the molecular sieve due to reduced pressure will enter the absorption column of the average pressure, reducing the purity of nitrogen products. Absorption time changes, the optimal average pressure time is basically the same, that is, the absorption time change has little effect on the optimal average pressure time.

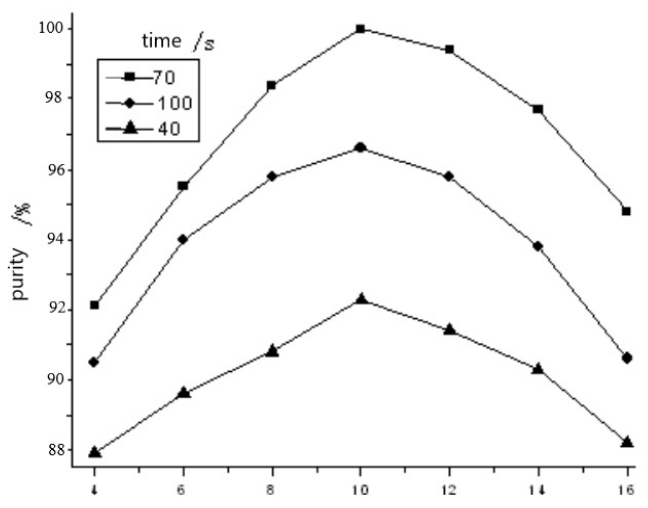

Figure 5 Effect of average pressure time on purity of oxygen products at different adsorption times

\section{4 conclusion}

The development and optimization of variable pressure adsorption nitrogen technology provides a reliable nitrogen supply for high purity nitrogen and coal mine underground nitrogen injection and fire suppression in low-concentration coal seam gas deep-cooled liquefaction devices. PSA technology will continue to improve as the demand for nitrogen increases in the coal mine sector. Through the experimental study of the adsorption performance of the device, the conclusions are as follows:

(1) there is an optimal adsorption time, The greater the intake, the shorter the adsorption time for the product to achieve optimal purity, when the air flow is large, the purity of the product is reduced.

(2) With the increase of adsorption pressure, product purity gradually increased, when the adsorption pressure 
reached $0.46 \mathrm{MPa}$, and then increase the pressure, nitrogen purity has a tendency to decrease; Adsorption pressure has a greater impact on product purity and recovery rate at low blowback times than when blowback time is high.

(3) There is an optimal average pressure time, adsorption time change on the optimal average pressure time effect is not obvious.

\section{References}

1. Liu Jianzhong,Sun Haitao,Lei Yi. New technology for coal seam gas development and utilization in coal mining areas[J]. coal industry , 2020, 45(01):258-267.

2. Chen Jinhua,Xiao Lu,Ling Hulei. Study on the deep cold liquefaturization process of low-concentration coal seam gas $[\mathrm{J}]$. coal technology,2016, 44 (6): 134-139, 181.

3. Yang Senjie. Effect of mixture refrigerant composition on natural gas light hydrocarbon recovery ethane process $[\mathrm{J}]$. China's petroleum and chemical standards and quality, 2019, 039(015):187-188.

4. Xiao Lu, Yao Chenglin. The gas source adaptability test of the low concentration coal seam gas liquefast separation device was studied[J]. coal industry, 2017(01):246-252.

5. Xiao Lu,Gan Hailong. The exhaust gas utilization of PSA nitrogen machine in low-concentration coal seam gas deep-cooled liquefaturization device is discussed[J]. Mining safety and environmental protection, 2017, 44(002):107-110.

6. Wang Changyuan,Sunwu,Xiong Yunwei. Study on the test parameters of oxygen-containing liquefied cold box of low-concentration coal seam gas[J]. Mining safety and environmental protection, 2017, 44(002):107-110.

7. Lu Kechao,Long Feifei. The pressure-absorbing nitrogen device is optimized for modification[J]. rock technology;, 2019, 26(007):186-187.

8. Miao Xuedong,Zhang Siwen,Xiao Yufei. The application of variable pressure adsorption oxygen-active molecules in flue gas denitrification engineering of biomass power plants[J]. gas separate, 2019. 